\title{
ChemComm
}

\section{High charge carrier mobility and efficient charge separation in highly soluble perylenetetracarboxyl-diimides $\uparrow$}

Cite this: Chem. Commun., 2014, 50,4955

Received 15th January 2014, Accepted 21st March 2014

DOI: $10.1039 / c 4 c c 00330 f$

www.rsc.org/chemcomm
D. Deniz Günbaş, $\ddagger^{\mathrm{a}}$ Chenming Xue $\ddagger^{\mathrm{b}}$ Sameer Patwardhan, ${ }^{a}$ Maria C. Fravventura, ${ }^{a}$ Hao Zhang, ${ }^{b}$ Wolter F. Jager, ${ }^{c}$ Ernst J. R. Sudhölter, ${ }^{c}$ Laurens D. A. Siebbeles, ${ }^{a}$ Tom J. Savenije, ${ }^{a}$ Shi Jin ${ }^{b}$ and Ferdinand C. Grozema*a
In this communication we report on the synthesis and charge mobility of highly soluble perylenebisimid derivatives. We show that introduction of alkylester side chains results in compounds combining a high solubility with charge mobilities up to $0.22 \mathrm{~cm}^{2} \mathrm{~V}^{-1} \mathrm{~s}^{-1}$. These materials are therefore interesting as an electron acceptor for solution-processed organic photovoltaics.

Perylene-3,4,9,10-tetracarboxylic acid diimides (PDIs) are n-type organic semiconducting materials that are attractive candidates for application in devices such as field effect transistors ${ }^{1,2}$ and photovoltaic cells. ${ }^{3-5}$ One of the key advantages of using organic materials for such devices is the possibility of depositing the materials in large areas from solution, for instance by spin coating. This will not only make processing considerably cheaper than for conventional inorganic solar cells but also offer the possibility of exploiting selfassembly properties that can be programmed into the individual molecules. ${ }^{6}$ A combination of excellent solubility in common organic solvents with good charge transport characteristics is therefore an important requirement for organic semiconductors. The majority of synthetic efforts and structure property studies have been devoted to p-type semiconductors, while the overall development of n-type materials is still lagging behind their p-type counterparts. ${ }^{1}$ Among the n-type organic semiconductors, PDIs have attracted a lot of attention due to their high fluorescence quantum yield, high photochemical stability and strong electron accepting properties.

The charge transport properties of PDIs depend, to a large extent, on the crystalline ordering and molecular orientation. ${ }^{7-10}$ Therefore, controlling these parameters is a critical aspect of

\footnotetext{
${ }^{a}$ Optoelectronic Materials, Department of Chemical Engineering,

Delft University of Technology, Julianalaan 136, Delft, The Netherlands. E-mail: f.c.grozema@tudelft.nl; Tel: +31152783914

${ }^{b}$ Department of Chemistry, City University of New York, College of Staten Island, 2800 Victoria Boulevard, Staten Island, NY 10314, USA

${ }^{c}$ Organic Materials and Interfaces, Department of Chemical Engineering, Delft University of Technology, Julianalaan 136, Delft, The Netherlands $\dagger$ Electronic supplementary information (ESI) available. See DOI: 10.1039/ c4cc00330f

\$ These authors have contributed equally to the work presented.
}

improving their performance in applications. For PDIs, substitution at the bay area and imide nitrogens has been employed to enhance their n-type character and to modify the molecular packing in the crystal. Charge carrier mobilities as high as $0.1 \mathrm{~cm}^{2} \mathrm{~V}^{-1} \mathrm{~s}^{-1}$ have been achieved upon substitution with strongly electron withdrawing groups in the bay area of the perylene core, despite their twisted perylene skeletons. ${ }^{11,12}$ However, it has been shown that high charge carrier mobilities for N-substituted PDIs are often combined with rather poor solubility.

In this communication, we address the lack of solubility of $\mathrm{N}$-substituted PDIs by reporting the properties of PDIs in which ester groups are incorporated into the aliphatic chains that are attached at the $\mathrm{N}$ atom of the imide functionality. To gain a better understanding of the relationship between the location of the ester group on the aliphatic unit and the charge transport characteristics, we have synthesized a series of PDIs (1-8) featuring alkyl ester $\mathrm{N}$-substituents. Our results show that the solid-state packing and charge transport properties can be tuned by varying the relative position of the ester group in the side chain with respect to the perylene core. Moreover, the potential of these highly soluble PDIs with high charge mobility for organic photovoltaics is demonstrated by presenting efficient charge generation in blends with a p-type $\mathrm{Cu}(\mathrm{II})$ phthalocyanine substituted with eight dodecyloxy chains in the peripheral positions (9), see Fig. 1.

PDIs 1-8 were synthesized by using a modification of a previously described procedure. ${ }^{13,14}$ The solubility of these PDI derivatives reflects the strength of intermolecular interactions

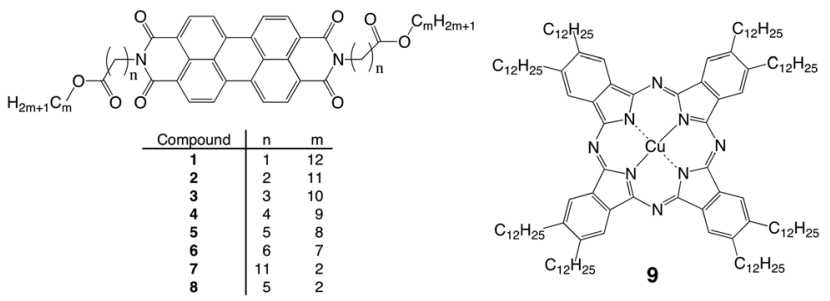

Fig. 1 Chemical structures of PDIs (1-8) and octa-dodecyloxysubstituted $\mathrm{CU}(॥)$ phthalocyanine (CUPc) 9 
in the solid state, which can be influenced by both the $\pi-\pi$ stacking of the perylene moieties and packing of the N-substituents. The only difference among the PDIs (except 8) is the position of the ester group in the PDI side chain. It is well known that ester groups can engage in a relatively strong dipole-dipole attractive interactions that can be probed by infrared spectroscopy since this results in a red shifted $\mathrm{C}=\mathrm{O}$ stretching frequency $\left(\nu_{\mathrm{C}}=\mathrm{O}\right) \cdot{ }^{15}$ Such an ester dipole-dipole interaction may hinder the efficient packing of all-anti polymethylene segments, leading to a weakened overall $\mathrm{N}$-substituent intermolecular interaction.

This could result in a higher solubility without sacrificing the PDI $\pi$-stacking interaction. Such an effect of ester groups on the packing of polymethylene segments has been observed to result in a considerably reduced melting temperature of a series of $n$-alkyl alkanate ester isomers compared to their $n$-alkane counterpart, see ESI. $\dagger$ As shown by the $\nu_{\mathrm{C}=\mathrm{O}}$ values in Table S1 (ESI $\dagger$ ), ester groups in PDIs 1-8 engage in dipole-dipole interactions, although to a different degree. The ester group in $\mathbf{1}$ exhibits the strongest dipole-dipole interaction, as indicated by the largest red-shift relative to the estimated "free" ester $\nu_{\mathrm{C}=\mathrm{O}}$ (Table 1 and Fig. S7, ESI $\dagger$ ). However, because the ester group is located near the end of the $\mathrm{N}$-substituent, it imposes only a small effect on the packing of polymethylene chains. Consequently, $\mathbf{1}$ features mainly all-anti $n$-alkyl chains and the lowest solubility, as shown in Table 1. Typically, all-anti polymethylene sequences exhibit asymmetric $\mathrm{CH}_{2}$ stretching at or below $2920 \mathrm{~cm}^{-1}$, whereas the introduction of gauche bonds results in a shift to higher frequencies. As the ester group moves towards the centre of the chain, the effect imposed by ester groups on the tight packing of the all-anti polymethylene chains becomes larger, resulting in strongly increased solubilities. In PDIs 5 and 6 the ester group is near the centre of the $\mathrm{N}$-substituent, giving them the highest solubility among the series studied. The ester group in 7 is close to the end of the N-substituent. Therefore, its effect on polymethylene chain packing becomes weaker, which translates into a lower solubility than for 5 and $\mathbf{6}$. This observed relation between the solubility and the ester position is qualitatively the same as the relation between the melting point and the ester position exhibited by a series of $n$-alkyl alkanate isomers. The fact that $\mathbf{1}$ and 2, and even 3 show much lower solubilities than 7 suggests that when the ester group is close to the PDI core, the ester dipole-dipole interaction and PDI $\pi-\pi$ stacking interaction may work cooperatively, leading to stronger intermolecular interaction and therefore a lower solubility. This reinforcing effect diminishes quickly with the

Table 1 Solid and "free" $\nu_{\mathrm{C}=\mathrm{O}}$, RT solubility and charge carrier mobilities $(\Sigma \mu)$ at $20{ }^{\circ} \mathrm{C}$ for compounds $\mathbf{1 - 8}$

\begin{tabular}{lllcl}
\hline $\begin{array}{l}\text { Solid } \nu_{\mathrm{C}=\mathrm{O}} \\
\left(\mathrm{cm}^{-1}\right)\end{array}$ & $\begin{array}{l}\text { "free" } \\
\left(\mathrm{cm}^{-1}\right)\end{array}$ & $\begin{array}{l}\nu_{\mathrm{C}=\mathrm{O}} \\
\text { Polubility }\left(\mathrm{mg} \mathrm{mL} \mathrm{CHCl}_{3} \text { at RT }\right.\end{array}$ & $\begin{array}{l}\Sigma \mu \\
\left(\mathrm{cm}^{2} \mathrm{~V}^{-1} \mathrm{~s}^{-1}\right)\end{array}$ \\
\hline $\mathbf{1}$ & 1730 & 1765 & 0.03 & 0.03 \\
$\mathbf{2}$ & 1740,1721 & 1749 & 0.12 & 0.06 \\
$\mathbf{3}$ & 1743 & 1746 & 0.62 & 0.17 \\
$\mathbf{4}$ & 1716 & 1745 & 84 & 0.05 \\
$\mathbf{5}$ & $1729,1734,1742$ & 1744 & 105 & 0.11 \\
$\mathbf{6}$ & 1732 & 1744 & 100 & 0.12 \\
7 & 1726,1734 & 1744 & 48 & 0.10 \\
$\mathbf{8}$ & 1728,1738 & 1745 & 86 & 0.22
\end{tabular}

increased separation between the PDI core and the ester group. It is intriguing to observe that the solubility changes by more than three orders of magnitude just by changing the position of the ester group in the N-substituent. Since aliphatic hydrocarbons do not make a sizeable contribution to the electric conductivity, one would like to minimize their usage as long as a good solubility can be achieved. PDI 8 was synthesized as the more "atom-efficient" version of $\mathbf{5}$, with only a modest decrease in solubility.

$\mathrm{X}$-ray diffraction (XRD) experiments were carried out to probe the influence of the ester groups on the molecular packing of the PDI molecules. The powder diffraction patterns shown in Fig. 2 illustrate sharp diffraction peaks indicating a high crystallinity for all PDIs except 7, which appears to be relatively disordered, as indicated by the broadest diffraction peaks.

The intensity of the small-angle diffractions relative to diffraction peaks around $2 \theta=20^{\circ}$ that usually arise from the packing of $n$-alkyl chains depends on the electron density contrast between the rigid core and aliphatic portions. Among highly soluble PDIs, the weakest small angle-diffraction has been observed for $\mathbf{4}$, while PDI 5 and $\mathbf{6}$ exhibit the strongest small-angle diffraction. This may be attributed to highly ordered PDI cores and/or poorly packed polymethylene chains as both lead to an increase in the PDI core/aliphatic chain electron density contrast. The latter is confirmed by FTIR data and a broad wide-angle diffraction at about $2 \theta=20^{\circ}$. Furthermore, sharp wide-angle peaks in $\mathbf{5}$ and $\mathbf{6}$ point to a highly ordered arrangement of PDI cores. In contrast, wide-angle

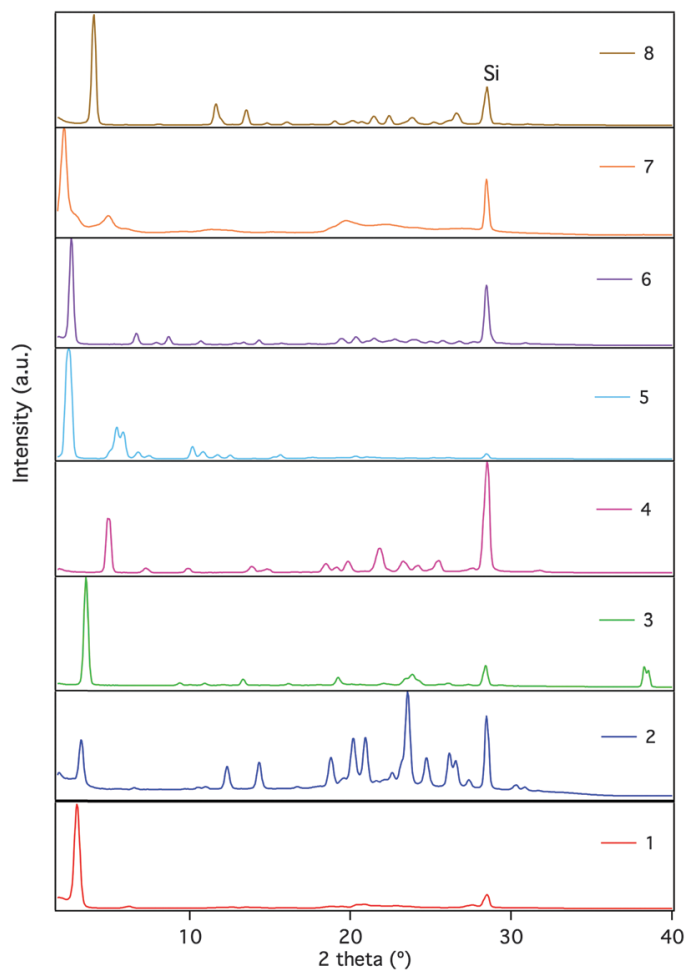

Fig. 2 Wide angle X-ray diffraction patterns of solid PDIs (1-8) Si: the diffraction from silicon powder, which was added as the internal calibration. The broad scattering from disordered alkyl chains was shown as dashed curves. 
diffraction of 4 does not display any broad scattering near $2 \theta=20^{\circ}$, suggesting well-ordered side chains. A comparison between 1 and $\mathbf{4}$ reveals that $\mathbf{1}$ gives much stronger small-angle diffraction than $\mathbf{4}$, even though their side chains are also closely packed in crystalline fashion. Therefore, in PDI 4 the optimal packing of the side chains presumably dominates the self-assembling properties, while stacking of PDI cores is weak.

The charge transport properties in powders of PDIs 1-8 were explored using the pulse-radiolysis time-resolved microwave conductivity (PR-TRMC) technique. ${ }^{16}$ It has been shown that mobilities obtained by this technique are similar to the optimal values from time-of-flight ${ }^{17}$ or field effect transistor ${ }^{18}$ measurements. The PR-TRMC technique can therefore be considered a method of choice for the fast and reproducible screening of charge transport properties of organic materials, before incorporation into devices. Charges are generated homogeneously in the samples by exposing them to a highly energetic, pulsed electron beam. PR-TRMC transients of 1-8 are depicted in Fig. 3 and the sum of the charge carrier mobilities determined from the end of pulse conductivity, $\Sigma \mu$, are compiled in Table 1 . The mobility values range from $0.03 \mathrm{~cm}^{2} \mathrm{~V}^{-1} \mathrm{~s}^{-1}$ for 1 to $0.22 \mathrm{~cm}^{2} \mathrm{~V}^{-1} \mathrm{~s}^{-1}$ for 8 . The highest one-dimensional mobility achieved for $8\left(0.22 \mathrm{~cm}^{2} \mathrm{~V}^{-1} \mathrm{~s}^{-1}\right)$ is in accordance with the values obtained for closely related $\mathrm{N}$-substituted PDIs measured using the same technique. ${ }^{19,20}$ It is worth noting that we were able to obtain PDIs with high charge carrier combined with excellent solubility. Among PDI isomers (1-7), 3 exhibits the highest mobility. This suggests that the position of the ester group in the side chains leads to stacking geometries that are favourable for charge transport in 3 . $^{13}$ PDI 4 shows the lowest mobility among highly soluble PDIs (4-7), which can be attributed to the weaker $\pi-\pi$ stacking interaction of PDI cores as suggested by the XRD results.

A comparison of the PR-TRMC conductivity transients demonstrates dramatic difference in the lifetimes of the charge carrying species for the PDI derivatives. The first half-lifetime in 8 is less than 20 ns, whereas PDI 7 has a half-lifetime of $43 \mu \mathrm{s}$. The increased lifetime of the charge carriers in 7 is attributed to a combination of enhanced morphology and molecular structure. The length of the side chains has a marked influence on the morphology and can strongly affect the recombination if this occurs by tunneling between neighbouring PDI stacks or layers through the hydrocarbon sidechains. ${ }^{21}$ In fact, the spacing between

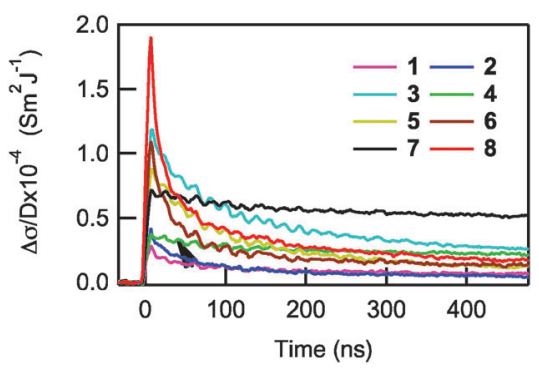

Fig. 3 Microwave conductivity transients of pure PDIs (1-8) obtained by exposing samples to 10 ns of $3 \mathrm{MeV}$ electrons generated using a Van de Graaf accelerator. two adjacent PDI stacks/layers in 7 is indeed the largest, as shown by the small-angle region of XRD results.

The high solubility of the compounds discussed here, combined with relatively high charge mobilities, makes them especially interesting for solution-processed organic photovoltaic cells. This is true in particular for PDI 7 that combines a high charge carrier mobility $\left(\sim 0.1 \mathrm{~cm}^{2} \mathrm{~V}^{-1} \mathrm{~s}^{-1}\right)$ with a very long conductivity half-life time. This indicates that one of the important factors that limit the overall efficiency of organic solar cells, trapping of charges, is unimportant in this material.

In order to explore the potential of PDI 7 as an acceptor material for application in organic photovoltaics, we have investigated the charge separation in bulk heterojunctions of PDI 7 blended with a p-type organic material, alkoxy-substituted $\mathrm{Cu}(\mathrm{II})$ phthalocyanine, see Fig. 1. The optical absorption spectra of individual layers of 7 and 9 together with a layer of CuPc 9: PDI 7 blend in a 50:50 weight ratio are shown in Fig. 4B. AFM images (see ESI $\dagger$ ) show an irregular surface, indicating the presence of extensive domains of at least one of the constituents. The generation of mobile charges in these three layers was studied using the photo-induced time-resolved microwave conductance (TRMC) technique. ${ }^{22,23}$ In this electrodeless photoconductivity technique, the formation of mobile charges on excitation with a nanosecond laser pulse is detected by monitoring the absorption of microwaves as a function of time. Details of the measurements are summarized in the ESI. $\dagger$

In Fig. 4A TRMC traces of the CuPc 9:PDI 7 blend $(50: 50)$ films and the pure materials, normalized by their optical attenuation $\left(F_{\mathrm{A}}\right)$, are shown. The samples were excited at $500 \mathrm{~nm}$
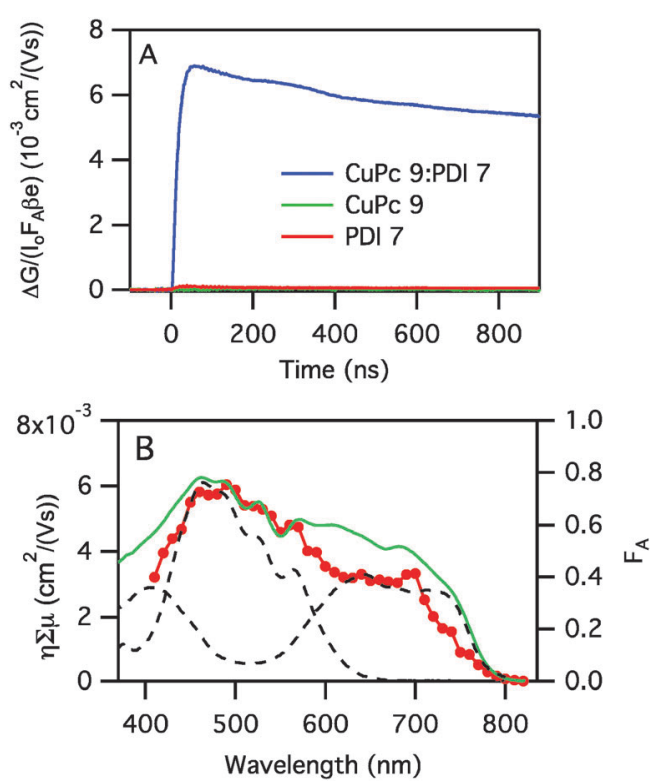

Fig. 4 (A) Photoconductance transients obtained upon excitation at $500 \mathrm{~nm}$ for pure PDI 7, pure CuPc 9 and 50:50 (wt\%) CuPc 9:PDI 7 blend films. (B) The product of the yield per incident photon and the sum of the charge carrier mobilities (red, left axis) and the fraction of the absorbed light $\left(F_{\mathrm{A}}\right)$ (green, right axis) in CuPc 9:PDI 7 blend film as a function of the excitation wavelength. The scaled spectra of films of the individual compounds are also included. 
with a fluence of $8.9 \times 10^{12}$ photons per $\mathrm{cm}^{2}$ per pulse. The photoinduced conductance increases upon photoexcitation due to the generation of mobile charge carriers. Subsequently, a decrease in photoconductance is observed as a result of charge recombination and/or trapping at defect sites. ${ }^{24}$ The rise time is determined by the laser pulse duration (3.5 ns) and the response time of the microwave cavity $(18 \mathrm{~ns}) .^{22}$ For the CuPc 9:PDI 7 blend the maximum in photoconductance is determined to be $8.0 \times$ $10^{-3} \mathrm{~cm}^{2} \mathrm{~V}^{-1} \mathrm{~s}^{-1}$, which is two orders of magnitude higher than those for the individual layers. This clearly shows that long-lived, mobile charge carriers are efficiently formed in the CuPc 9:PDI 7 blend upon excitation at $500 \mathrm{~nm}$, despite the fact that relatively large domains are present. The long lifetime is consistent with the presence of these domains in which electrons and holes can move around freely.

An efficient solar cell should take advantage of the excellent spectral coverage due to the complementary optical absorption of PDIs and phthalocyanines (see Fig. 4B). This requires that efficient generation of charges should occur, regardless of the excitation wavelength. In bulk heterojunctions of PDI 7 and CuPc 9 the photocurrent can be generated in two ways: (1) photoexcitation of PDI $(\sim 500 \mathrm{~nm})$ followed by hole transfer to the CuPc phase; (2) photoexcitation of CuPc $(\sim 700 \mathrm{~nm})$ followed by electron transfer to the PDI phase. The result in both cases is an electron in the LUMO of the PDI and a hole in the HOMO of the CuPc.

In Fig. 4A the product of the fraction of incident photons that leads to charge separation $(\eta)$ and the sum of the mobility of the positive and negative charges $(\Sigma \mu)$, derived from the maximum photoconductance for the CuPc 9:PDI 7 blend is shown as a function of excitation wavelength.

The value of $\eta \Sigma \mu$ is compared with the fraction of the incident light that is absorbed in a blend film, $F_{\mathrm{A}}$. Since $\Sigma \mu$ does not depend on the way in which the charges were generated; this comparison shows that the quantum yield for charge generation, $\varphi$, corresponding to excitation of 9 is similar to excitation of 7 .

In conclusion, we have described the synthesis and characterization of highly soluble PDI derivatives (1-8) that exhibit charge carrier mobilities up to $0.22 \mathrm{~cm}^{2} \mathrm{~V}^{-1} \mathrm{~s}^{-1}$. The potential of these materials for application in solutionprocessed organic photovoltaics was demonstrated by blending PDI 7 with a p-type phthalocyanine derivative. Photoconductance measurements on this blend show the efficient formation of free charges on absorption of light in both components of the blend.

This work is part of the Joint Solar Programme (JSP) of the Foundation for Scientific Research on Matter (FOM), which is part of the Netherlands Organization for Scientific Research (NWO). The research leading to these results has received funding from the European Research Council under the European Union's Seventh Framework Programme (FP7/2007-2013)/ERC Grant agreement no 240299. The authors are grateful to the NSF for funding the acquisition of the SAXS/WAX system through award CHE-0723028. They also acknowledge the partial support of this research by Donors of the American Chemical Society Petroleum Research Fund.

\section{Notes and references}

1 Y. Wen and Y. Liu, Adv. Mater., 2010, 22, 1331-1345.

2 F. Würthner and M. Stolte, Chem. Commun., 2011, 47, 5109-5115.

3 L. Schmidt-Mende, A. Fechtenkötter, K. Müllen, E. Moons, R. H. Friend and J. D. Mackenzie, Science, 2001, 293, 1119-1122.

4 V. Kamm, G. Battagliarin, I. A. Howard, W. Pisula, A. Mavrinskiy, C. Li, K. Mullen and F. Laquai, Adv. Energy Mater., 2011, 1, 297-302.

5 M. Guide, S. Pla, A. Sharenko, P. Zlar, F. Fernandez-Lazaro, A. SastreSantos and T.-C. Nguyen, Phys. Chem. Chem. Phys., 2013, 15, 18894-18899.

6 F. Würthner, Chem. Commun., 2004, 1564-1579.

7 M. Ruiz Delgado, E.-G. Kim, D. A. da Silva Filho and J.-L. Bredas, J. Am. Chem. Soc., 2010, 132, 3375-3387.

8 J. Vura-Weiss, M. A. Ratner and M. R. Wasielewski, J. Am. Chem. Soc., 2010, 132, 1738-1739.

9 V. Marcon, W. Pisula, J. Dahl, D. W. Breiby, J. Kirkpatrick, S. Patwardhan, F. C. Grozema and D. Andrienko, J. Am. Chem. Soc., 2009, 131, 11426-11432.

10 F. May, V. Marcon, M. R. Hansen, F. C. Grozema and D. Andrienko, J. Mater. Chem., 2011, 21, 9538-9545.

11 R. Schmidt, J. H. Oh, Y. S. Sun, M. Deppisch, A. M. Krause, K. Radacki, H. Braunschweig, M. Konemann, P. Erk, Z. A. Bao and F. Würthner, J. Am. Chem. Soc., 2009, 131, 6215-6228.

12 B. A. Jones, M. J. Ahrens, M.-H. Yoon, A. Facchetti, T. J. Marks and M. R. Wasielewski, Angew. Chem., Int. Ed., 2004, 43, 6363-6366.

13 C. Xue and S. Jin, Chem. Mater., 2011, 23, 2689-2692.

14 Y. J. Xu, S. W. Leng, C. M. Xue, R. K. Sun, J. Pan, J. Ford and S. Jin, Angew. Chem., Int. Ed., 2007, 46, 3896-3899.

15 S. Hesse and M. A. Suhm, Phys. Chem. Chem. Phys., 2009, 11, 11157-11170.

16 J. M. Warman, M. P. de Haas, G. Dicker, F. C. Grozema, J. Piris and M. G. Debije, Chem. Mater., 2004, 16, 4600-4609.

17 A. M. van de Craats, J. M. Warman, M. P. de Haas, D. Adam, J. Simmerer, D. Haarer and P. Schuhmacher, Adv. Mater., 1996, 8, $823-826$.

18 B. Wegewijs, M. P. de Haas, D. M. de Leeuw, R. Wilson and H. Sirringhaus, Synth. Met., 1999, 101, 534.

19 C. W. Struijk, A. B. Sieval, J. E. J. Dakhorst, M. van Dijk, P. Kimkes, R. B. M. Koehorst, H. Donker, T. J. Schaafsma, S. J. Picken, A. M. van de Craats, J. M. Warman, H. Zuilhof and E. J. R. Sudholter, J. Am. Chem. Soc., 2000, 122, 11057-11066.

20 Z. J. Chen, V. Stepanenko, V. Dehm, P. Prins, L. D. A. Siebbeles, J. Seibt, P. Marquetand, V. Engel and F. Wurthner, Chem. - Eur. J., 2007, 13, 436-449.

21 J. M. Warman, J. Piris, W. Pisula, M. Kastler, D. Wasserfallen and K. Müllen, J. Am. Chem. Soc., 2005, 127, 14257-14262.

22 J. E. Kroeze, T. J. Savenije, M. J. W. Vermeulen and J. M. Warman, J. Phys. Chem. B, 2003, 107, 7696-7705.

23 T. J. Savenije, A. J. Ferguson, N. Kopidakis and G. Rumbles, J. Phys. Chem. C, 2013, 117, 24085-24103.

24 T. J. Savenije, D. H. K. Murthy, M. Gunz, J. Gorenflot, L. D. A. Siebbeles, V. Dyakonov and C. Deibel, J. Phys. Chem. Lett., 2011, 2, 1368-1371. 